\title{
Visiones políticas y perspectivas de Mercosur, CAN y Unasur en la construcción de una ciudadanía regional (2002-2016)
}

\author{
Political views and perspectives of Mercosur, \\ CAN and Unasur in the construction of a regional \\ citizenship (2002-2016)
}

María Gabriela Rho ${ }^{a *}$ (DD https://orcid.org/0000-0003-0959-2877
Recibido el 17 de agosto de 2018. Aceptado el 17 de octubre de 2018. Publicado el 22 de octubre de 2018.

*Autor para correspondencia: María Gabriela Rho, correo electrónico: mgabrielarho@gmail.com

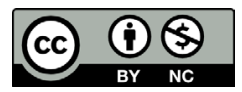

Esta obra está protegida bajo una Licencia Creative Commons Atribución-NoComercial 4.0 Internacional.

\begin{abstract}
a Universidad Nacional de Córdoba y Consejo Nacional de Investigaciones Científicas y Técnicas, becaria doctoral en el Centro de Investigaciones y Estudios sobre Cultura y Sociedad, Argentina, correo electrónico: mgabrielarho@gmail.com
\end{abstract}

\section{Resumen}

El objetivo de este trabajo es analizar, desde una perspectiva comparada, las visiones políticas y lineamientos adoptados por el Mercado Común del Sur (Mercosur), la Comunidad Andina de Naciones (CAN) y la Unión de Naciones Sudamericanas (Unasur), en relación con las propuestas de construcción de ciudadanía en la región sudamericana entre los años 2002 y 2016. La metodología de investigación es de carácter cualitativa y se centra en el análisis de documentos públicos oficiales, emitidos por dichos organismos de integración regional, que se enmarcan dentro de lineamientos en clave de ciudadanía regional. La relevancia del artículo radica en la reflexión respecto a qué tipo de ciudadano y qué concepción de ciudadanía se desprende de los lineamientos impulsados por can, Mercosur y Unasur. En este sentido, muestra que, bajo la categoría de ciudadanía, se esconden una multiplicidad de estatus legales que sirven como dispositivos de selección, jerarquización e inclusión diferencial de los migrantes regionales.

Palabras clave: ciudadanía, Mercosur, can, Unasur.

\section{Abstract}

The aim of this work is to analyze, from a comparative perspective, the political views and guidelines adopted by the Southern Common Market (Mercosur), the Andean Community of Nations (CAN), and the Union of South American Nations (Unasur) in relation to the proposals put forward to construct citizenship in the South American region between 2002 and 2016. A qualitative research methodology is used, and it is focused on the analysis of official public documents issued by the above mentioned organizations of regional integration, which follow a set

CÓMO CITAR: Rho, M. G. (2018). Visiones políticas y perspectivas de Mercosur, can y Unasur en la construcción de una ciudadanía regional (2002-2016) [Political views and perspectives of Mercosur, CAN and Unasur in the construction of a regional citizenship (2002-2016)]. Estudios Fronterizos, 19, e015. doi:https://doi.org/10.21670/ref.1815015 
of guidelines in terms of regional citizenship. The importance of the paper lies in the reflection upon the kind of citizen and the concept of citizenship that derives from the guidelines promoted by CAN, Mercosur, and Unasur. In this sense, it shows that a multiplicity of legal status hides under the category of citizenship, and that they serve as selection, hierarchization and differential inclusion mechanisms for regional migrants.

Keywords: citizenship, Mercosur, CAN, Unasur.

\section{Introducción}

En la región sudamericana, a comienzo del siglo xxi, se producen transformaciones significativas en las lógicas, visiones y discursos políticos con que tradicionalmente se venían definiendo las políticas migratorias. A diferencia del discurso político dominante de los años noventa, articulado con base en la "retórica de la exclusión", ${ }^{1}$ se configura una nueva manera de abordar la cuestión migratoria bajo una "retórica de la inclusión", cimentada en la perspectiva de los derechos humanos, la ciudadanía comunitaria y el pluralismo cultural (Domenech, 2009). Así, en varios países se derogan leyes migratorias adoptadas durante las dictaduras militares que se sustentaban en visiones punitivas y securitistas que criminalizaban y estigmatizaban a los migrantes; y se promueven nuevas leyes o anteproyectos que reconocen nuevos derechos a las poblaciones migrantes; se abre el debate respecto a los derechos que fueron marginados en las agendas de los Estados nacionales y los organismos de integración regionales, como son los derechos políticos y culturales y, surgen nuevos movimientos y organizaciones sociales de migrantes que comienzan a debatir en torno a las nuevas políticas migratorias.

Estas transformaciones también se hacen visibles en los lineamientos impulsados por los organismos de integración sudamericanos: Comunidad Andina de Naciones (CAN), ${ }^{2}$ Mercado Común del Sur (Mercosur) ${ }^{3}$ y Unión de Naciones Suramericanas (Unasur), ${ }^{4}$ los cuales, comienzan a elaborar propuestas políticas de construcción de una ciudadanía regional. Si bien, dichos proyectos presentan matices significativos en

\footnotetext{
${ }^{1}$ La "retórica de la exclusión" entiende a las migraciones regionales como un "problema" y amenaza al empleo, las condiciones sanitarias, al orden público y a la integración territorial, por lo que se plasma en mecanismos de control de fronteras y residencia restrictivos y coercitivos, así como en prácticas de carácter persecutorio y represivo en relación con la presencia migrante (Domenech, 2009, p. 26). Mármora (2010, p. 76) inscribe este tipo de retórica dentro de lo que denomina modelo de la "securitización", el cual diseña las políticas migratorias como parte de la seguridad nacional de los Estados nación.

${ }^{2}$ La CAN se conformó en el año 1969 mediante la firma del Acuerdo de Cartagena por parte de Bolivia, Colombia, Ecuador, Perú y Chile. Chile se retiró en el año 1976. Venezuela se incorporó en el año 1973 y se retiró en el año 2006. Actualmente, los países miembros son: Bolivia, Ecuador, Colombia y Perú. Como países asociados participan Argentina, Brasil, Chile, Paraguay y Uruguay (CAN, s.f.).

${ }^{3}$ El Mercosur se fundó en el año 1991 a través de la firma del Tratado de Asunción por Argentina, Brasil, Paraguay y Uruguay. Participan como estados asociados: Chile, Colombia, Ecuador, Guyana, Perú y Surinam. Venezuela se adhiere al tratado constitutivo en el año 2006 y actualmente se encuentra suspendida como Estado parte. Bolivia se incorpora en el año 2015 y se encuentra en vías de incorporación (Mercosur, s.f.c).

${ }^{4}$ En el año 2004 en la Reunión de Presidentes de América del Sur se creó la Comunidad Suramericana de Naciones (CSN) que, en el año 2007, pasaría a llamarse Unasur. Se encuentra formada por los 12 países sudamericanos: Argentina, Bolivia, Perú, Ecuador, Colombia, Venezuela, Chile, Brasil, Paraguay, Uruguay, Surinam y Guyana (Unasur, s.f.).
} 
relación con los derechos que establecen para los migrantes regionales, los requisitos que se solicitan para tramitar la residencia, el alcance de los acuerdos y las formas de conceptualizar la ciudadanía regional, creemos que, en términos generales, esbozan nuevas formas de adhesión e "inclusión" de los migrantes regionales a los Estados nación. En un contexto caracterizado por la configuración de un régimen global de control de las migraciones que ya no apunta a la exclusión de los migrantes sino a canalizar, valorizar y explotar los elementos de excedente de los movimientos migratorios (Mezzadra, 2005, p. 148), es importante problematizar los códigos de inclusión y los dispositivos de selección, jerarquización y estratificación que los lineamientos de cAN, Mercosur y Unasur establecen, para así poder reflexionar respecto a qué tipo de ciudadano y qué concepción de ciudadanía se desprende.

Es así que, en el presente trabajo interesa analizar, desde una perspectiva comparada, los lineamientos y las visiones políticas adoptadas por dichos organismos de integración en relación con las propuestas de construcción de ciudadanía regional. En primer lugar, se realizará una aproximación al contexto global y regional que enmarca y habilita el impulso de dichos lineamientos, por lo que, se introducirá el régimen global de control de las migraciones y su reconfiguración en el espacio sudamericano a partir de la crisis de legitimidad política del neoliberalismo. En segundo lugar, se analizarán los lineamientos de CAN, Mercosur y Unasur enmarcados en las propuestas políticas de construcción de una ciudadanía regional, con el objetivo de identificar los puntos de acuerdo y articulaciones, así como las tensiones y divergencias, que se presentan entre los lineamientos elaborados por los organismos de integración regional. Por último, se propone reflexionar de manera crítica sobre las visiones y perspectivas que sustentan los lineamientos en clave de ciudadanía regional de can, Mercosur y Unasur.

Para esto se utiliza una estrategia metodológica de carácter cualitativo, centrada en el análisis de documentos públicos oficiales, como son el Acuerdo $\mathrm{N}^{\circ}$ 14/02 Residencia para nacionales de los Estados partes del Mercosur, Bolivia y Chile (2002); el Estatuto de la Ciudadanía del Mercosur (2010); la Decisión 1343 Estatuto Andino de Movilidad Humana (2015); y, el Informe Conceptual sobre Ciudadanía Sudamericana (2014) ${ }^{5} \mathrm{El}$ análisis de los documentos se encuentra guiado por la perspectiva teórica y metodológica de la historia social comparada, la cual permite estudiar procesos políticos, sociales y económicos cercanos en el tiempo y en el espacio que, al encontrarse sujetos a la acción de las mismas causas e influirse mutuamente, comparten una serie de elementos y problemas específicos (Devoto, 2004). El método comparativo se utilizará para analizar las semejanzas como para indagar en las diferencias que se dan en procesos similares, en un mismo momento histórico (Bulcourf y Cardozo, 2008, p. 17). De este modo, la comparación de los lineamientos impulsados por los organismos de integración regional se realizará a partir de la categoría de ciudadanía regional, identificando cómo se enuncian los derechos políticos, sociales y culturales; los requisitos para tramitar la residencia; los elementos vinculados a la securitización de las migraciones y los marcos institucionales en los que se inscriben los acuerdos.

\footnotetext{
${ }^{5}$ Del mismo modo, se consultaron documentos resultantes de las reuniones de los foros especializados sobre migraciones de CAN y Mercosur, como así también, de las actividades del Grupo de Trabajo sobre Ciudadanía Suramericana (GTCS) de la Unasur, como son: Primer Foro Andino de Migraciones (CAN, 2008); Segundo Foro Andino de Migraciones (CAN, 2009); Tercer Foro Andino de Migraciones (CAN, 2012); Cuarto Foro Andino de Migraciones (CAN, 2013); Información sobre las actividades del GTCS de la Unasur (2013); Memoria Institucional Del Foro Especializado Migratorio del Mercosur y Estados Asociados (Mercosur, s.f.b).
} 


\section{Las políticas de gobernabilidad migratoria: Una visión desde el espacio político sudamericano}

Desde mediados de los años noventa, la emergencia de un régimen global de control de las migraciones instala una nueva manera de organizar, clasificar y controlar los movimientos internacionales de población (Domenech, 2013, p. 6). Este régimen es una adaptación de las lógicas empresariales y de gestión que organismos internacionales, como el Banco Mundial y el Fondo Monetario Internacional, estaban delineando para materializar los programas de reestructuración neoliberal del Estado; por lo que, para el campo de las migraciones, es promovido por agencias multilaterales que forman parte de estas estructuras hegemónicas del sistema mundial, como la Organización Internacional para las Migraciones (OIM) y Naciones Unidas (Estupiñán, 2013, pp. 8-9).

Una variedad importante de reformas en materia de legislación y políticas migratorias, inscriptas en este régimen, encuentran su sustento ideológico en la perspectiva de la gobernabilidad migratoria o migration management (Domenech, 2013, p. 2). La perspectiva de la gobernabilidad migratoria parte del diagnóstico de la inevitabilidad de las migraciones y la inutilidad de las medidas restrictivas para contenerlas, por lo que busca crear e implementar modelos y políticas que permitan administrar, ordenar y controlar de manera eficaz los flujos migratorios con el fin de encauzarlos, dentro de marcos normativos claramente delineados, hacia objetivos y demandas que apunten a la maximización de beneficios y rentabilidad económica de la mano de obra migrante (Estupiñán, 2013; Geiger y Pécoud, 2012). Es así que, este enfoque se presenta como una alternativa menos "violenta" para controlar las migraciones, ya que no se pretende "cerrar" herméticamente las fronteras y establecer mecanismos explícitos de coerción, sino establecer un sistema de dique para producir un proceso activo de inclusión del trabajo de los migrantes a través de procesos de regulación (Mezzadra, 2005, p. 148).

Por ello, la idea de "migración ordenada" se apoya en una lógica costo-beneficio de clasificación de los migrantes que, sobre la base de su supuesta utilidad o no, establece una serie de "ventajas-beneficios" o "desventajas-perjuicios" en relación con lo que los migrantes pueden "aportar" al desarrollo económico, la reducción de la pobreza, así como al "enriquecimiento cultural" (Domenech, 2008, p. 58). Con el propósito de obtener la legitimidad necesaria para su instrumentación y lograr mayores resultados en la administración eficaz de las migraciones, la perspectiva de la gobernabilidad migratoria se apoya en el discurso de los derechos humanos de los migrantes. Pero, estas políticas si bien se enmarcan en el amparo y defensa de los derechos humanos, desplazan (no eliminan) mecanismos y formas de regulación y control restrictivas y coercitivas con las cuales conviven (Domenech, 2013, p. 2).

La perspectiva de la gobernabilidad desplaza los códigos de integración social debido a que desdibuja y torna borrosas las líneas entre la inclusión y la exclusión. Los lineamientos y políticas migratorias enmarcadas en este enfoque promueven un proceso de accesibilidad desigual a varias zonas e instituciones de la sociedad ya que reconocen algunos derechos propios de los ciudadanos (sobre todo aquellos vinculados al mercado laboral) pero, al mismo tiempo, restringen el acceso a otros (derechos políticos y de participación), lo que supone una inclusión diferencial que incluye excluyendo a los migrantes de las estructuras de los Estados nación (Mezzadra y Neilson, 2016, p. 249). De este modo, la inclusión de los migrantes nunca es completa ya que se encuentra sujeta a diferentes grados de subordinación, comando, 
discriminación y segmentación y se convierte en una forma de control y disciplina, que sirven para seleccionar y filtrar personas y sus diferentes modos de circulación, en modos no menos violentos de aquellos empleados por las prácticas de expulsión (Mezzadra y Neilson, 2016, pp. 245 y 379).

Esta superposición de múltiples líneas de inclusión y exclusión produce la estratificación y multiplicación de los sistemas de ingreso y residencia de los migrantes, surgiendo una multiplicidad de estatus que tienden a explotar el perfil unitario de la ciudadanía (Mezzadra y Neilson, 2016, pp. 253 y 387). En la actualidad, esta no se presenta como una categoría monolítica y unitaria sino que se da lugar a un proceso de "gradación de la ciudadanía”, es decir, se configuran diferentes y gradados "tipos de ciudadanos" en términos de acceso a derechos, encontrándose los migrantes en el medio de una escala entre ciudadanos "plenos" y los que se consideran "no-ciudadanos" (Varela, 2015, pp. 275-277). Por lo que, la categoría de inclusión diferencial intenta captar el sentido de la crisis de la figura unitaria del ciudadano y la correspondiente producción de múltiples condiciones de ciudadanía (Mezzadra y Neilson, 2016, p. 245).

En la región sudamericana la perspectiva de la gobernabilidad migratoria se arraiga a comienzos de la década del 2000 con la inauguración de la Conferencia Sudamericana de Migraciones (CSM) ${ }^{6}$ La CSM se convierte en un espacio privilegiado de diálogo y coordinación de políticas migratorias entre los países sudamericanos, lo que da impulso a un proceso de regionalización de las políticas migratorias, generando mayores grados de compatibilización y convergencia en los lineamientos impulsados por los organismos de integración estudiados. ${ }^{7}$ Sin embargo, la perspectiva de la gobernabilidad migratoria no se trata necesariamente de una visión integral y unívoca, ya que su desarrollo difiere según los intereses de los actores políticos implicados, así como, de las especificidades del contexto sociohistórico en las que se arraiga (Domenech, 2013, p. 5). En la región sudamericana, en el periodo analizado, se acentúan aquellos elementos vinculados a la defensa de los derechos humanos y se impulsan lineamientos y políticas orientados a la regularización de los migrantes, como también, se incorpora la categoría de ciudadanía para enmarcar la ampliación de derechos en el marco de los organismos de integración regional.

Esto se debe a que, con la crisis de legitimidad del neoliberalismo se abre un nuevo escenario político regional con la emergencia de gobiernos de centro izquierda y progresistas que promueven una serie de transformaciones en el plano político y social, que impactan y tienen efectos en el discurso político, con lo que se comienza a abordar la cuestión migratoria. Nos referimos, por ejemplo, a la recuperación de la capacidad reguladora del Estado respecto al mercado; la mayor participación de los sectores populares en la construcción del Estado y el fortalecimiento de las

\footnotetext{
${ }^{6}$ Con el objetivo de consolidar la visión global de gobernabilidad de las migraciones la oIM conforma Procesos Consultivos Regionales de Migración (PCR), como espacios no vinculantes de discusión, socialización de políticas, coordinación y validación de posicionamientos y enfoques comunes sobre migraciones donde intervienen Estados, organismos internacionales y representantes de la sociedad civil organizada (Santi, 2011).

${ }^{7}$ A partir de 2006 se observa un doble proceso en los mecanismos de diálogo que se establecen entre los organismos de integración regional y la CSM: por un lado, los acuerdos alcanzados en la CSM empiezan a ser considerados puntos centrales y las bases para avanzar en debates al interior de los foros migratorios o de los lineamientos impulsados por CAN, Mercosur y Unasur. Y, por otro lado, en miras a tener una mayor capacidad de incidencia y poder de negociación en la CSM, Mercosur, CAN y Unasur comienzan a elaborar agendas de trabajo para proponer en las reuniones de la CSM, pasando a ocupar un papel activo en el planteo de temas por tratar, así como en las orientaciones de los lineamientos consensuados.
} 
organizaciones sociales; el impulso de políticas de inclusión y redistribución de ingreso tendientes a recomponer las condiciones de vida de los sectores medios y populares; el restablecimiento de derechos laborales e incremento de los niveles de empleo, entre otras. Del mismo modo, estos gobiernos inician una serie de rupturas en las formas de comprender la configuración de los organismos de integración, impulsando un doble proceso: por un lado, CAN y Mercosur realizan un "giro hacia lo social" a nivel discursivo e institucional, es decir, buscan profundizar los procesos de integración ampliando sus agendas y marcos de acuerdos hacia el tratamiento de cuestiones sociales y culturales (Romano, 2009, p. 258). Por otro lado, surgen nuevas propuestas de integración alternativas como la Unasur que, al priorizar una agenda política sobre la económica, amplían los marcos de cooperación en ámbitos no comerciales, cobrando mayor relevancia las dimensiones sociales e incorporando nuevos temas que fueron marginados en los años noventa (Sanahuja, 2012).

Este nuevo escenario político regional es lo que permite replantear y revisar los lineamientos y visiones en torno a las migraciones y los migrantes por parte de los organismos de integración estudiados y así, reconfigurar de manera singular la perspectiva de la gobernabilidad migratoria. Con las transformaciones en los discursos y políticas sociales impulsadas por estos gobiernos y la configuración de los organismos de integración regional, se habilitó un escenario favorable para que comenzaran a cambiar las visiones y perspectivas con las que se abordaba la cuestión migratoria. Como se señaló, es en este contexto que surgen lineamientos enmarcados en propuestas políticas de construcción de una ciudadanía regional, analizados a continuación.

\section{Mercosur y el Estatuto de la Ciudadanía}

El Estatuto de la Ciudadanía del Mercosur. Plan de acción (2010), se presenta como un proyecto elaborado desde una perspectiva multidimensional de la integración, es decir, que se propone contemplar acciones y medidas en materia política, económica, comercial, social, educativa, de cooperación judicial y de seguridad. En este sentido, a partir de un lenguaje que enfatiza el discurso de los derechos humanos, plantea profundizar la dimensión social y ciudadana del proceso de integración.

Tal como lo señala su nombre, el objetivo de este lineamiento es establecer un plan de acción para conformar, de manera progresiva, un estatuto de ciudadanía, el cual debería estar integrado por un conjunto de derechos fundamentales y beneficios para los migrantes regionales. Así, se plantea avanzar en la implementación de una política de libre circulación de personas en la región; igualar los derechos y libertades civiles, sociales, culturales y económicas para los nacionales de los Estados parte e igualar las condiciones de acceso al trabajo, salud y educación (Estatuto de la Ciudadanía del Mercosur. Plan de Acción, 2010, art. 2). Para alcanzar dichos objetivos se delimitan 11 ejes que deben ser abordados por diferentes órganos y grupos de trabajo del Mercosur: Circulación de personas; Fronteras; Identificación; Documentación y cooperación consular; Trabajo y empleo; Previsión social; Educación; Transporte; Comunicaciones; Defensa del consumidor y Derechos políticos.

En relación al desarrollo de estos ejes, aquellos vinculados a cuestiones de fronteras, documentación, trabajo, previsión social, transporte y educación, el Estatuto no realiza un planteo estructural y renovado en su tratamiento, en tanto se reduce 
a compilar y proponer la revisión y fortalecimiento de lineamientos que Mercosur viene impulsando y debatiendo desde su conformación en la década del noventa. ${ }^{8}$ De este modo, si contemplamos la totalidad de los ejes propuestos y el enfoque a partir del cual son abordados, podemos decir que el Estatuto continúa otorgándole gran importancia a cuestiones vinculadas a seguridad y control de las fronteras y limita el alcance de los lineamientos a facilitar la circulación de profesionales, estudiantes de nivel superior, turistas, trabajadores cualificados o aquellos que poseen un trabajo formal. En este sentido, no se contemplan ni se amplían las agendas al tratamiento de cuestiones y problemáticas que afectan al grueso de los migrantes regionales.

El único elemento novedoso que añade el Estatuto de Ciudadanía (2010) es el debate en torno a los derechos políticos de los migrantes regionales. La incorporación de esta dimensión es significativa debido a que hasta el momento no se encontraba abordada de manera articulada en ámbitos regionales, aunque a nivel de las legislaciones nacionales los doce países de Sudamérica permiten a los residentes votar en elecciones locales (municipales). Así, el artículo 11 establece que se van a:

Evaluar las condiciones para avanzar progresivamente en el establecimiento de derechos políticos, de acuerdo con las legislaciones nacionales que reglamenten su ejercicio, en favor de los ciudadanos de un Estado parte de Mercosur que residen en otro Estado Parte del que no son nacionales, incluyendo la posibilidad de elegir parlamentarios del Mercosur (Estatuto de Ciudadanía, 2010, p. 4).

Una primera observación es que no se desarrolla qué se entiende por derechos políticos y, con base en lo expuesto, pareciera ser que se limitaran a la participación electoral. Es necesario advertir que, el derecho al voto para migrantes es lo más visible en cuanto a derechos políticos, pero que hay que incorporar al debate otra serie de derechos como el de asociarse, reunirse con fines políticos, acceder a cargos públicos, etc. (Ramírez, 2016). En segundo lugar, se desprende que el planteo es de carácter sumamente exploratorio y su debate se encuentra condicionado por lo reglamentado en las legislaciones nacionales; por lo que, el abordaje queda reducido a elaborar un estado de situación sin especificar ni avanzar en mecanismos concretos que permitan la efectiva ampliación de derechos políticos para los migrantes por fuera de lo que ya se encuentra establecido.

Un elemento llamativo del Estatuto de la Ciudadanía es la nula mención a derechos culturales. Esta omisión es significativa debido a que en la etapa en la cual fue impulsado, a nivel regional, se adoptan discursos afines al pluralismo cultural para abordar las políticas migratorias. Sin embargo, se puede recuperar para nuestro análisis lo planteado en la Cartilla de la Ciudadanía del Mercosur (Mercosur, s.f.a). ${ }^{9}$ En esta se observa cómo la dimensión cultural implicada en los procesos de migración es entendida como un problema de "integración" planteándose como objetivos la ampliación y

\footnotetext{
${ }^{8}$ Como ejemplos se pueden señalar, la implementación de las Áreas de Control Integrado; la armonización de las informaciones para la emisión de documentos de identificación en los Estados Partes; la ampliación de los mecanismos de cooperación consular; la revisión del Acuerdo de Recife y de la Declaración Sociolaboral (1998); profundizar el Sistema de Acreditación Regional de Carreras Universitarias (Sistema ARCU-SUR) y los mecanismos para la simplificación de los trámites administrativos a efectos de la equivalencia de estudios y títulos de enseñanza superior; entre otros.

${ }^{9}$ La Cartilla es impulsada en el año 2014 y se encuentra en formato en línea. En ella, se han recopilado y sistematizado las principales normas vigentes de interés para los migrantes regionales y se han incluido los organismos responsables de la aplicación de aquellas en cada Estado parte o asociado.
} 
el fortalecimiento del intercambio cultural entre los ciudadanos del Mercosur y la cooperación entre instituciones y agentes culturales a fin de favorecer programas y proyectos culturales conjuntos (Mercosur, s.f.a). De este modo, los lineamientos quedan reducidos a la generación de canales de intercambio de información, limitados a espacios académicos y especializados sobre determinadas culturas, mediante la puesta en marcha de proyectos institucionales que permitan "conocer" e identificar la diversidad de identidades culturales existentes en la región. ${ }^{10}$

Esta forma de entender los procesos de integración no se compromete más allá de ciertas prácticas enmarcadas en el "modelo de comprensión cultural". El mismo se apoya en una visión de consenso y en un discurso de aceptación, tolerancia y respeto de las diferentes culturas, que apunta, a nivel práctico, a mejorar la comunicación entre los diferentes grupos étnicos y fomentar cambios de actitudes para revertir los prejuicios, focalizando sus propuestas en programas de sensibilización y formación de relaciones humanas (McCarthy, citado en Domenech y Magliano, 2008, p. 435). Esta forma de abordar el pluralismo cultural entiende a la "diversidad cultural" como no conflictiva y no jerárquica, lo que supone la coexistencia armónica y horizontal de una variedad de grupos y formas culturales. Así, reconoce a la sociedad como multicultural y multiétnica, valora la "contribución" de los grupos étnicos al desarrollo del país y promueve el respeto a la diversidad cultural y el reconocimiento de derechos de igualdad formal, pero mantiene inalterada y no cuestiona la estructura de poder que reproduce las condiciones materiales y simbólicas de desigualdad y exclusión social (Domenech, 2007, pp. 25 y 32).

Por último, más allá del Estatuto de la Ciudadanía (2010), se considera que para comprender los lineamientos del Mercosur en esta etapa es necesario recuperar en nuestro análisis el Acuerdo № 14/02 Residencia para Nacionales de los Estados Partes del Mercosur, Bolivia y Chile, impulsado en el año 2002. ${ }^{11}$ Dicho Acuerdo es una de las líneas de trabajo prioritarias del Foro Especializado Migratorio del Mercosur (FEM) ya que, su plena aplicación y vigencia, es el interés principal de la mayoría de las reuniones. ${ }^{12}$ Del mismo modo, es el modelo y la base sobre el que se van a pensar mecanismos de regularización y residencia de personas en la región sudamericana y, sobre el cual, la CAN se va a apoyar para formular lineamientos migratorios. En este sentido, será el principal instrumento de articulación y convergencia de políticas migratorias entre CAN y Mercosur ya que, todos los países miembros de la CAN, a excepción de Venezuela, comienzan a adherir e implementarlo a partir de 2011, proceso que da impulso, desde el año 2013, al trabajo coordinado entre ambos organismos de integración regional.

\footnotetext{
${ }^{10}$ Esto se visualiza en los objetivos de Mercosur restringidos a la "creación de espacios culturales y la realización de acciones que expresen las tradiciones históricas, los valores comunes y la diversidad de los ciudadanos" (Mercosur, s.f.a, p. 81), así como en las acciones que plantea llevar adelante, siendo reducidas al "intercambio de artistas, escritores, investigadores, grupos artísticos e integrantes de entidades vinculadas a la cultura" (Mercosur, s.f.a, p. 81); a favorecer producciones audiovisuales bajo el régimen de co-producción y co-distribución, así como la cooperación entre los archivos históricos y a la formación común de recursos humanos involucrados en facilitar la circulación de material destinado a la realización de eventos culturales (Mercosur, s.f.a).

${ }^{11}$ El Acuerdo №14/02 sobre residencia, pese a que se impulsó en el año 2002, entró en vigencia hasta el año 2009, debido a que Argentina, Brasil y Bolivia lo aprobaron en el año 2004, Uruguay y Chile durante el año 2005 y, por último, Paraguay en el año 2008.

12 El FEM es un espacio que funciona en el ámbito de las Reuniones de Ministros de Interior de Mercosur y Estados Asociados. Se crea en el año 2003 y se reúne de manera periódica.
} 
El Acuerdo № 14/02 tiene como objetivo establecer un área de libre circulación y residencia de personas en el espacio mercosureño y se centra en establecer mecanismos de regularización de los migrantes regionales. ${ }^{13}$ Para esto establece dos tipos de residencia, una temporaria y otra permanente. El primer tipo de residencia se otorga por un periodo de dos años y para adquirirla los solicitantes deben presentar: certificado de identidad; partida de nacimiento y comprobación de estado civil; la carencia de antecedentes penales, judiciales y policiales internacionales, en el país de origen y de recepción; certificado médico en caso que lo exijan y pagar una tasa retributiva de servicios (Acuerdo $\mathrm{N}^{\circ} 14 / 02,2002$, art. 4). Para el caso de la residencia permanente se deben presentar los mismos requisitos y además, la constancia de residencia temporaria y la acreditación de medios de vida lícitos que permitan la subsistencia (Acuerdo $\mathrm{N}^{\circ} 14 / 02,2002$, art. 5).

Se observa que si bien el Acuerdo $\mathrm{N}^{\circ} 14 / 02$, por un periodo de dos años, otorga un tipo de residencia que no exige poseer un trabajo formal para establecerse en otro país, termina restringiendo la capacidad de residir a la cuestión laboral. Los criterios de selección que operan se asientan en criterios instrumentalistas y economicistas ya que los migrantes, para obtener la residencia permanente, deben demostrar su "utilidad" y una adecuada inserción a los mercados de trabajo. A su vez, al solicitar la carencia de antecedentes penales, encontramos la pervivencia de elementos del modelo de securitización debido a que se sigue pensando al migrante como una amenaza potencial a la seguridad del país de destino.

Es necesario prestar atención a estos criterios de selección que establece el acuerdo $\mathrm{N}^{\mathrm{o}} 14 / 02$ ya que van a permear los debates y la concepción de ciudadanía presente en los lineamientos del Mercosur, así como del resto de los lineamientos impulsados en la región. Por lo anterior, se puede decir que el Estatuto de la Ciudadanía del Mercosur no logra superar lo planteado por el Acuerdo $\mathrm{N}^{\circ} 14 / 02$ sobre residencia. La categoría de ciudadanía es incorporada de forma discursiva y simbólica ya que el estatuto no realiza un planteo estructural y renovado para avanzar en la conformación de una ciudadanía mercosureña. El acuerdo $\mathrm{N}^{\circ} 14 / 02$, no solo es el eje de trabajo prioritario al interior del Mercosur, sino que además se convierte en un instrumento de suma relevancia para la articulación y coordinación de políticas migratorias en la región sudamericana, por lo que es central para comprender las lógicas, perspectivas y límites de los posteriores proyectos de construcción de ciudadanía.

\section{El Estatuto Andino de Movilidad Humana de la Comunidad Andina de Naciones}

La CAN comienza a impulsar lineamientos en clave de ciudadanía a partir del año 2008, cuando se crea el Foro Andino Migratorio (FAM).$^{14}$ Dicho espacio de debate

\footnotetext{
${ }^{13}$ Es necesario señalar que el Acuerdo № 14/02 (2002) se enmarca en el lenguaje de los derechos humanos ya que amplía e incorpora una serie de derechos a los migrantes regionales, como son: el libre tránsito (interno y externo); el derecho a la reunificación familiar y a un trato igualitario con los nacionales en relación con la legislación laboral, condiciones de trabajo, seguros sociales y aportes previsionales; el derecho a transferir remesas al país de origen; a que los hijos de los migrantes puedan acceder a una nacionalidad y a las instituciones de educación, independientemente de su situación migratoria (art. 9).

${ }^{14}$ El FAM no se reúne de manera periódica sino que, durante el periodo analizado se realizaron cuatro encuentros del mismo en los años 2008, 2009, 2012 y 2013.
} 
dinamiza nuevas perspectivas para abordar temáticas migratorias, las cuales cristalizan en la aprobación, en el año 2015, de la Decisión 1343 Estatuto Andino de la Movilidad Humana; ${ }^{15}$ el cual, es una propuesta normativa que reúne y sistematiza los avances alcanzados respecto a la movilidad humana con el objetivo de lograr la armonización y unificación de normas regionales (Decisión 1343, 2015, p. 16). Para los fines de este trabajo, se puede dividir en dos grandes bloques: los capítulos que establecen derechos, deberes y obligaciones de los ciudadanos andinos y aquellos que refieren a las modalidades para el ejercicio de la movilidad humana. ${ }^{16}$

Respecto al primer bloque, la Decisión 1343 parte del reconocimiento del derecho a migrar y plantea una serie de derechos generales para los migrantes regionales como son: la no discriminación, igual trato que a los nacionales, la libre movilidad, no deportación sin un procedimiento justo, la conservación de sus documentos de identidad, la no privación de su libertad por el hecho de encontrarse en situación irregular, la reunificación familiar y, el acceso a derechos políticos, a la participación, la salud y la educación (Decisión 1343, 2015, sección primera, capítulo 2). Asimismo, señala de forma particular derechos que se establecen para grupos de población especialmente vulnerable —niños, género y víctimas de conflicto armado interno o de violencia generalizada- (sección segunda, capítulo 2); para solicitantes de asilo o refugio, víctimas de trata y/o tráfico de personas (sección tercera, capítulo 2); derechos vinculados a las relaciones de trabajo (sección cuarta, capítulo 2) y derechos culturales de pueblos andinos (sección quinta, capítulo 2). A su vez, el Estatuto cambia el término "migración" por el de "movilidad humana", el cual busca abarcar todos los procesos de traslado de un sitio diferente al de residencia habitual contemplando la existencia de múltiples razones que ocasionan dicha movilidad (Decisión 1343, 2015, p. 15), como pueden ser, "procesos de emigración, inmigración, retorno, solicitud de asilo y protección internacional, desplazamientos internos y reasentamientos" (Ramírez, 2016, p. 51).

En términos generales se puede decir que, hasta el momento, no encontramos ningún lineamiento en la región que presente niveles tan altos de sistematización e integralidad al momento de plantear derechos para los migrantes. A diferencia del Estatuto de la Ciudadanía del Mercosur, la Decisión 1343 presenta un instrumento de sistematización novedoso, que muestra una perspectiva renovada en el tratamiento de problemáticas migratorias y refuerza elementos vinculados a la defensa y ampliación de derechos humanos.

No obstante, si se examinan las condiciones que se establecen para obtener la residencia, encontramos una fuerte sintonía con lo propuesto por Mercosur. Al igual que en el Acuerdo $\mathrm{N}^{\circ}$ 14/02 sobre residencia se estipula una residencia temporal y otra permanente. En el primer caso, el permiso es de dos años y se debe presentar:

\footnotetext{
${ }^{15}$ Se sostiene que este Estatuto forma parte de una nueva manera de abordar la cuestión migratoria ya que presenta transformaciones significativas en relación con la Decisión 545 Instrumento Andino de Migración Laboral (2003), la cual no solo no logra ser reglamentada sino que además, propone espacios de libre circulación limitados a trabajadores en relación de dependencia, por lo que, los derechos que establece se reducen a aquellos vinculados a cuestiones laborales.

${ }^{16}$ Se puede mencionar otro eje que aborda la Decisión 1343 y que, para los fines de este trabajo, se decidió no incorporar su análisis: migración extraregional, protección consular a los ciudadanos andinos en situación de movilidad fuera de la región. En el mismo se pautan acciones tendientes a la cooperación consular a aquellos nacionales de los países miembros de la CAN que no cuentan con representación consular de su país de origen en la localidad en que se encuentran.
} 
documento de identificación; declaración jurada de inexistencia de procesos penales activos iniciados en su contra, o en cualquier otro país que no sea el de su nacionalidad por delitos relacionados con la trata de personas, tráfico de migrantes, narcotráfico o pornografía infantil; y la acreditación de fondos suficientes para solventar gastos durante su permanencia en el país (Decisión 1343, 2015, capítulo 4, art. 41, Permiso de residencia temporal).${ }^{17}$ Para obtener la residencia permanente debe presentarse la constancia de autorización de residencia temporaria; documento de identidad; justificativo que dé cuenta de la permanencia en el territorio de al menos dos años y justificativo del carácter lícito de los fondos y actividades desarrolladas por el ciudadano andino (Decisión 1343, 2015, capítulo 4, art. 44, Residencia permanente).

A través de estos criterios, la Decisión 1343 (2015) no logra superar la vinculación entre migración, trabajo y residencia. Si bien, el Acuerdo $N^{\circ}$ 14/02 de Mercosur abre la posibilidad de residir en la región por un periodo de dos años sin la necesidad de acreditar "medios de vida lícitos", en la Decisión 1343 de la can este es un criterio que es exigido desde el momento que se solicita la residencia temporaria. El requisito de poseer un justificativo bajo declaración jurada de la licitud de los fondos o de la actividad laboral desarrollada, excluye al grueso de los trabajadores migrantes que cuentan con empleos informales o no reconocidos como tales.

A su vez, del mismo modo que el Acuerdo $\mathrm{N}^{\circ}$ 14/02, se exige la condición de certificar la inexistencia de antecedentes penales. Sin embargo, la diferencia fundamental reside en que son contemplados exclusivamente los denominados delitos mayores, es decir, se limita el derecho a circular y residir en la región únicamente a aquellas personas que tengan antecedentes por delitos vinculados al "tráfico de migrantes, trata de personas, pornografía infantil o narcotráfico" (Decisión 1343, 2015, art. 35, p. 52). El Acuerdo $\mathrm{N}^{\circ} 14 / 02$ sobre residencia, al no especificar a qué tipos de delitos se refiere -enunciando de forma ambigua "carencia de antecedentes judiciales y/o penales y/o policiales" (Acuerdo № 14/02, 2002, art. 4, p. 4) - supone que prohíbe el ingreso y la obtención de la residencia a cualquier persona que cuente con alguno de estos antecedentes, así sean delitos menores o una simple contravención como, por ejemplo, los que se derivan de situaciones como la venta en la vía pública, vivir en una casa o terreno tomado, el corte de una calle, etcétera.

Respecto a los derechos políticos, la Decisión 1343 establece que "los Estados Miembros promoverán la participación política de los ciudadanos andinos que hayan establecido su residencia en otro país de la Comunidad Andina" (Decisión 1343, 2015, p. 30). En este punto se considera importante realizar dos comentarios. Por un lado, se encuentran fuertes limitaciones debido a que, si bien se señala que los migrantes regionales "gozan de todos los derechos establecidos para los ciudadanos nacionales" (Decisión 1343, 2015, p. 34), se aclara que estos pueden verse condicionados por "las limitaciones establecidas en las legislaciones internas de los Países Miembros en cuanto a los derechos relacionados con la participación política y el acceso a cargos o funciones en la administración pública” (Decisión 1343, 2015, p. 34). Por otro lado, los

\footnotetext{
${ }^{17}$ Para el caso de la solicitud de residencia temporal se establecen artículos diferenciados para permisos de residencia a estudiantes (Decisión 1343, 2015, art. 42) y trabajadores, compañeros permanentes y cónyuges (Decisión 1343, 2015, art. 43). Los requisitos son los mismos, especificándose para cada caso los referidos a la solvencia económica y licitud de las actividades económicas desarrolladas. Por ejemplo, a los estudiantes se les pide un certificado bancario que dé cuenta de la disponibilidad de recursos suficientes; a los inversionistas el origen de los fondos que se pretenden invertir en el país y a los trabajadores la licitud de la actividad económica y laboral que se pretende desarrollar.
} 
derechos políticos y de participación son abordados desde una perspectiva restringida ya que la Decisión 1343 se compromete solamente a generar las posibilidades de votar en procesos electorales en el exterior. Así, se define que los países deberán asegurar que sus oficinas consulares "dispongan de la infraestructura necesaria para garantizar el derecho al voto y a participar de los mecanismos de democracia directa de los nacionales en el exterior" (Decisión 1343, 2015, p. 37), volviéndose a reiterar que, la participación política de los migrantes en el país de recepción queda condicionada por lo dispuesto en la legislación nacional (Decisión 1343, 2015, p. 37). De este modo, al igual que Mercosur, no se avanza de forma significativa y novedosa en la ampliación de derechos políticos ya que no se elabora una propuesta que tensione lo establecido en las legislaciones nacionales y que cuestione la perspectiva que reduce los derechos políticos a la participación electoral.

Por último, en relación al reconocimiento de derechos culturales, la Decisión 1343 (2015) establece dos objetivos: primero, garantizar el derecho a que los ciudadanos y ciudadanas andinas continúen viviendo conforme a sus costumbres y prácticas sociales, respetando sus manifestaciones culturales; segundo, promover la convivencia intercultural y la integración de las personas en situación de movilidad en la comunidad de destino (Decisión 1343, 2015, capítulo 2, sección quinta, art. 30). Así, se plantea que los Estados miembros deben fomentar procesos de intercambio cultural, cohesión social, promoción de la igualdad y fortalecimiento de la cultura andina. Para el desarrollo de estos objetivos se establecen una serie de derechos como los de conservar y desarrollar sus propias formas de convivencia y organización social, así como sus conocimientos colectivos, saberes ancestrales, vestimentas, símbolos y emblemas de su cultura (Decisión 1343, 2015, capítulo 2, sección quinta, art. 31). De este modo, la dimensión cultural es abordada, al igual que Mercosur, desde una perspectiva afín al pluralismo cultural. La serie de derechos que se establecen se reducen a garantizar políticas de reconocimiento cultural y remiten a representaciones culturales estáticas, que se limitan a garantizar condiciones para que los pueblos andinos conserven y desarrollen sus formas de organización social, conocimientos, vestimentas, entre otros, negando el conflicto y los procesos de jerarquización y exclusión social que se producen a partir de la identidad cultural de los migrantes.

\section{Unasur y la ciudadanía sudamericana}

Unasur forma parte de las iniciativas de integración que buscan instalarse en la región sudamericana como propuestas alternativas a la cAN y el Mercosur, es así que, los ejes de integración económica y comercial adquieren menor peso, ganando prioridad la elaboración de consensos respecto a problemáticas políticas y sociales. En este sentido, la dimensión migratoria se vuelve un objetivo central en su agenda de integración, siendo novedoso en sus planteamientos el hecho de que instala y vincula el abordaje de la cuestión migratoria a los procesos de construcción de una ciudadanía sudamericana.

Pese a que en las primeras Reuniones de Presidentes de América del Sur se mencionaba la importancia de los aspectos migratorios, es recién en la II Cumbre de Jefes de Estado de la Comunidad Sudamericana de Naciones, en el año 2006, que se plantea iniciar un camino "hacia la construcción de una ciudadanía sudamericana" (Unasur, 2006, p. 4). La centralidad que adquiere este eje de trabajo queda plasmada 
en su incorporación al Tratado Constitutivo de Unión de Naciones Sudamericanas (2011, p. 10), estableciendo entre sus objetivos "la consolidación de una identidad suramericana a través del reconocimiento progresivo de derechos a los nacionales de un Estado Miembro residente en cualquiera de los otros Estados Miembros, con el fin de alcanzar una ciudadanía sudamericana”. Sin embargo, hasta el año 2014, no encontramos claridad respecto a qué se entiende y cuáles son los elementos y variables que contemplaría una propuesta de ciudadanía para la región. Así, las declaraciones se limitan a establecer de forma muy general que, a través de la construcción de la ciudadanía sudamericana, se busca reconocer de forma progresiva derechos civiles, políticos, laborales y sociales para los nacionales de los Estados miembros, bajo un enfoque integral y comprensivo, haciendo referencia a la puesta en marcha de procesos de regularización migratoria y armonización de políticas en la región.

Por lo que, si bien Unasur es el primer organismo de integración regional sudamericano que incorpora la categoría de ciudadanía para abordar la dimensión migratoria, es recién en el año 2012, mediante la Decisión №8/2012 del Consejo de Jefas y Jefes de Estado, que comienza un proceso de debate, trabajo y creación de espacios institucionales con miras a la elaboración de lineamientos que conceptualicen y establezcan las dimensiones que contemplaría el proyecto de ciudadanía sudamericana. De este modo, a través de dicha Decisión, se decreta el inicio de la construcción de la ciudadanía sudamericana, dando prioridad a la dimensión migratoria (Decisión $\mathrm{N}^{\circ} 8 / 2012,2012$, art.1). Además, se crea el Grupo de Trabajo sobre Ciudadanía Sudamericana (GTCS), asignándole la tarea de elaborar una Hoja de Ruta y un informe conceptual que explore las diferentes dimensiones de la Ciudadanía Sudamericana teniendo en cuenta las experiencias subregionales, la normativa interna de los Estados miembros, así como los avances en los diferentes Consejos de Unasur que se encuentren vinculados con la temática (art. 2), con el objetivo de superar lo planteado en estas instancias e ir más allá de la convergencia de los lineamientos ya impulsados. El primer borrador del Informe Conceptual es presentado en el año 2013 y, luego de dos reuniones realizadas durante el año 2014, finalmente se aprueba en el GTcs la redacción final del Informe Conceptual (Ramírez, 2016, p. 79).

El Informe Conceptual sobre ciudadanía sudamericana parte de sistematizar los antecedentes que se desarrollaron en el GTCS, en los Consejos Sectoriales de Unasur y en experiencias preexistentes como ser Mercosur, CAN, La Comunidad del Caribe (Caricom) y la Conferencia Sudamericana de Migraciones (CSM); para luego pasar a abordar la propuesta de construcción de ciudadanía en la región y definir los lineamientos estratégicos con base en tres preguntas: “¿cuál es el concepto de ciudadanía que está implicada en los cimientos de la construcción propuesta por la Unasur?, ¿cuáles son los elementos que han de componerla?, y ¿qué estructura jurídicopolítica ha de sostenerla? (Informe Conceptual sobre Ciudadanía Sudamericana, 2014, p. 9). En este sentido, el Informe propone una definición de ciudadanía sudamericana entendida como una condición jurídica de acceso a derechos y obligaciones; una ampliación, no sustitutiva, de las ciudadanías nacionales y una condición de identidad y pertenencia a la región sudamericana. Así, los ciudadanos y ciudadanas sudamericanos tendrían derecho a: la libre movilidad y residencia dentro de la región; la consecución de la igualdad de derechos y libertades civiles, sociales, culturales y económicas en cualquiera de los países; la protección y defensa de sus derechos contra el racismo, la xenofobia y la discriminación y el abuso de autoridad, así como contra 
la criminalización; la convivencia, la inclusión sociolaboral, la participación ciudadana y la integración; y a peticionar ante los organismos que a tal fin formen parte de la estructura institucional de la Unasur, el reconocimiento, reivindicación y defensa de estos derechos, en cualquier circunstancia que así lo amerite (Informe Conceptual sobre Ciudadanía Sudamericana, 2014, inciso 3). Con base en esta definición, Unasur avanza en una discusión conceptual sobre la categoría de ciudadanía y propone mecanismos para la conformación de una ciudadanía de alcance regional.

En primer lugar, esto marca una diferencia con la can y el Mercosur que, si bien hablan en términos de ciudadanía regional, no explicitan los mecanismos ni cómo interactuarían los espacios nacionales con las instancias de los organismos de integración regional. Por lo que, sus lineamientos terminan reduciéndose al reconocimiento de algunos derechos y obligaciones y a establecer criterios para solicitar la residencia, limitando su aplicabilidad a la predisposición o voluntad política de los gobiernos de los países miembros de reglamentar e incorporar dichos lineamientos a los marcos de las normativas nacionales. En este sentido, la propuesta de Unasur admite diferentes niveles de membresía, proponiendo la constitución de una ciudadanía mixta (Ramírez, 2016, p. 81), es decir, que se vincule con un espacio ampliado constituido por todos los países que la componen, sin dejar de reconocer y reafirmar las ciudadanías nacionales. Así, establece que "no existe contraposición inicial o primaria entre la ciudadanía nacional y la ciudadanía suramericana" (Informe Conceptual sobre Ciudadanía Sudamericana, 2014, p. 5), constituyéndose la segunda como una ampliación, no sustitutiva, de la primera, en la cual "todos los ciudadanos de los Estados parte, incorporarían de manera gradual, flexible y progresiva nuevos derechos a ser ejercidos en el marco de la entidad política mayor, la Unasur, como ampliación de las ciudadanías nacionales" (Informe Conceptual sobre Ciudadanía Sudamericana, 2014, p. 5).

En relación con esto, se abre un debate en torno a la estructura jurídico-política que garantizaría esta doble membresía que supone la ciudadanía sudamericana, introduciendo la cuestión del alcance supranacional de Unasur. El Informe Conceptual (2014, p. 9) establece que, a pesar de que "el entramado institucional de Unasur está aún en construcción", su Tratado Constitutivo "prevé el desarrollo institucional requerido para el cumplimiento de los mandatos de los órganos competentes" (Informe Conceptual, 2014, p. 9), comprometiéndose a iniciar un análisis y debate sobre la institucionalidad regional a fin de poner en funcionamiento "organismos e instancias que garanticen que cada derecho ciudadano pueda ser reivindicado ante ellos" (Informe Conceptual, 2014, p. 9).

En un principio Unasur plantea la constitución de un espacio dealcance supranacional, es decir, la construcción y aceptación de normas e instituciones que impliquen la cesión de algunos aspectos de la soberanía nacional en aras a la conformación de una institucionalidad regional. Sin embargo, Vior (2013) y Serbin (2010) advierten que esta perspectiva choca, en la práctica, con el principio de soberanía nacional que articula la convivencia de los Estados sudamericanos. En este sentido, señalan que determinados factores se convierten en obstáculos formidables para avanzar en la conformación de un espacio supranacional de alta densidad y efectividad. Entre ellos, se mencionan: la baja institucionalidad de los acuerdos alcanzados; la centralidad que asumen los Estados como principales promotores de las iniciativas de integración en el marco de acuerdos intergubernamentales y, frecuentemente, interpresidenciales; la marcada politización de la agenda regional que obliga a constituir consensos en un contexto marcado por la 
fragmentación y por la aspiración de liderazgos divergentes; y por la participación de gobiernos poco dispuestos a sacrificar aspectos de su soberanía o de su interés nacional en compromisos regionales.

Asimismo, se puede sumar que si bien el proceso de conformación de la ciudadanía sudamericana se plantea en el marco de un espacio político ampliado, las ciudadanías nacionales $\mathrm{y}$, principalmente, la soberanía nacional, tienen aún un fuerte peso en las perspectivas de constitución de la misma. Se sostiene esto ya que ser "nacional" de algún Estado miembro, resulta el fundamento central para poder acceder a la ciudadanía sudamericana, y es sobre las legislaciones nacionales y los compromisos regionales asumidos por los Estados miembros que se avanzaría en la construcción de proyectos de ciudadanía en la región (Unasur, 2013). Por lo que Unasur es un proyecto de Estados nacionales que aún conservan el monopolio de la soberanía y la ciudadanía nacional para reconocer a los miembros de su comunidad política.

En segundo lugar, otro elemento que se desprende de la definición de ciudadanía sudamericana propuesta por Unasur, es la cuestión de la identidad. Así, el GTcS apuesta a instaurar "una concepción de ciudadanía sudamericana que trascienda el fundamental repertorio de derechos y obligaciones, para contemplar también un proceso de identidad (identificación) o de reconocimiento de un sentido de pertenencia común" (Informe Conceptual sobre Ciudadanía Sudamericana, 2014, p. 12). En este sentido, se entiende a la ciudadanía sudamericana como una categoría jurídico-política y socio-cultural (Ramírez, 2016, p. 81).

En relación con la categoría de identidad, el Informe Conceptual la define "fundamentalmente por lo que ya somos, lo que proyectamos ser como Región y por la forma cómo nos posicionamos frente a terceros países y regiones" (2014, p. 12), debido a que se considera que Sudamérica ha pasado de ser una referencia geográfica, a ser un espacio político. Así, por un lado, respecto a lo que ya somos se refuerza la idea de que la identidad sudamericana se encuentra definida por elementos como una historia compartida; lazos de solidaridad que se gestaron en los procesos de independencia, tradiciones y costumbres; culturas diversas, lenguas e idiomas y cosmovisiones. A su vez, se la presenta como representativa de un espacio político con "valores compartidos como la democracia, el estado de derecho, el respeto irrestricto a los derechos humanos y la consolidación de Sudamérica como zona de paz" (Informe Conceptual, 2014, p. 13). Por otro lado, en relación con lo que proyectamos ser, los esfuerzos de Unasur apuntan a acordar una serie de valores, normas e instituciones que, mediante el reconocimiento de derechos, generen un sentido de solidaridad regional, a fin de lograr la convivencia, cohesión, empatía y confianza mutua entre aquellos que se saben y reconocen como iguales en el acceso a derechos en la región (Informe Conceptual sobre Ciudadanía Sudamericana, 2014, inciso 3). Así, Unasur utiliza dos estrategias para lograr este "sello identitario": busca recuperar y reforzar algunos elementos políticos, históricos y culturales que se encuentran en el imaginario social sudamericano; e intenta crear lealtades a la comunidad política mediante el establecimiento de derechos y valores comunes.

Ahora bien, se considera que mediante la categoría de identidad se hace un uso simbólico instrumental, ligado a objetivos económicos y comerciales de Unasur. ${ }^{18} \mathrm{El}$

\footnotetext{
${ }^{18}$ Varela (2015, p. 281), recuperando a Stolcke (1994), señala cómo la Unión Europea utiliza el desarrollo de un sentido de cultura compartida y de identidad de objetivos como forma de apoyo ideológico a una unión económica y política capaz de tener éxito a nivel internacional.
} 
objetivo de conformarse en un sujeto geopolítico de peso en el mapa multilateral mundial y tener mayor gravitación y representación en los mercados y foros internacionales, convierte la constitución de una identidad sudamericana en un eje privilegiado y un elemento fundamental, siendo los procesos de construcción de ciudadanía en la región un terreno fértil para avanzar en este sentido. Del mismo modo, los esfuerzos apuntan a crear, construir e instalar una identificación con la región que no necesariamente representa o se encuentra instalada en el imaginario social sudamericano, antes que contemplar aspectos culturales e históricos en términos transformadores, no tensionando las pretensiones homogeneizantes que sustenta a la categoría hegemónica de ciudadanía presente en la conformación de los Estados sudamericanos.

Como correlación de esto, los derechos culturales se reducen a: promover programas de becas y movilidad académica regional; visibilizar y valorar la diversidad cultural y el aporte de los pueblos indígenas, afrodescendientes y comunidades étnicas; lanzar políticas regionales en materia de integración idiomática; establecer una agenda educativa común que promueva la interculturalidad; generar redes académicas y agendas de investigación conjuntas y, elaborar un calendario de conmemoraciones regionales (Informe Conceptual sobre Ciudadanía Sudamericana, 2014, inciso 4). De este modo, las acciones propuestas tienden a reforzar un proyecto de "unidad cultural", ahora ya no vinculado a los procesos hegemónicos de un Estado nación sino a un ámbito regional, que no pretende trascender la perspectiva asimilacionista de tolerancia y respeto de la diversidad cultural plasmada en las perspectivas afines al pluralismo cultural que desarrollábamos para los casos de la can y el Mercosur.

En tercer lugar, es importante señalar que aún existen algunas cuestiones que el proyecto de Ciudadanía Sudamericana no logra superar en relación con los lineamientos de CAN y Mercosur. Por un lado, no se plantea eliminar la exigencia de no poseer antecedentes penales, judiciales y policiales. Por otro lado, ni en el Informe Conceptual ni en las reuniones del GTCS, se aborda la cuestión de la participación y derechos políticos de los migrantes, produciéndose un total silenciamiento en relación con esta dimensión.

Por último, como ya se señaló, en el Informe Conceptual (2014) no se explicitan los requisitos o mecanismos que se emplearían para obtener la ciudadanía sudamericana ni se aclara ante que instancias se debería solicitar la misma ya que los lineamientos estratégicos que se esbozan son muy generales y no hacen referencia a estas cuestiones. Sin embargo, se encuentra un punto de inflexión entre los años 2015 y 2016 en los debates desarrollados al interior del GTCS. En estas reuniones se trabajó en torno a la elaboración de un acuerdo de residencia Unasur que contemple la simplificación administrativa del trámite para la obtención de la residencia (Ramírez, 2016, p. 84). Para esto se plantearon dos acciones, primero, se acordó que "los Estados miembros de la Unasur propenderán acciones tendentes a reducir los requisitos y documentos exigidos, particularmente en: certificado médico y certificado de medio de vida" (Unasur, 2016, p. 3); segundo, se asumió el compromiso de "avanzar hacia la reducción progresiva de los costos para la tramitación de la residencia" (Unasur, 2016, p. 3). Así, a diferencia de los lineamientos de la can y el Mercosur, Unasur mediante el compromiso de eliminar aquellos requisitos que son restrictivos para tramitar la residencia, pues no solo reduce algunos obstáculos que afectan a la mayoría de los migrantes sino que, además, comienza a abrir un escenario para cuestionar las perspectivas instrumentalistas que vinculan las migraciones con los mercados de trabajo de forma directa. 
Una problemática sobre la cual Unasur no profundiza en las reuniones del GTCS, se vincula con las instancias institucionales que garantizarían la ciudadanía sudamericana. Se percibe que los Estados nacionales son las únicas estructuras institucionales ante las que se puede apelar, no solo para solicitar y tramitar la residencia, sino también para reclamar y demandar la vulneración o violación de derechos. Hasta el momento, los lineamientos de Unasur no poseen efectos jurídicos en los países de la región ya que no logró transformarse en un espacio de carácter supranacional, por lo que los migrantes regionales se encuentran supeditados a leyes y procedimientos nacionales.

\section{Conclusiones}

El nuevo escenario político sudamericano que se abre con la crisis de legitimidad política del neoliberalismo explica la forma singular en que la perspectiva de la gobernabilidad migratoria es resignificada y reconfigurada por los organismos de integración. La modificación en las concepciones respecto a cómo entender los procesos de integración regional y las trasformaciones en los discursos y políticas sociales y culturales llevadas adelante por estos gobiernos generó un clima propicio, no solo para que la cuestión migratoria gane mayor visibilidad, sino además para que comiencen a cambiar las lógicas con que tradicionalmente se ha abordado. Así, se impulsan nuevas políticas y comienzan a circular nuevos discursos que enfatizan la defensa de los derechos humanos, se incluyen también, nuevos ejes de debate como son los derechos culturales y políticos de los migrantes regionales. En este contexto, los organismos de integración regional estudiados encuadran la ampliación de derechos bajo la noción de ciudadanía, por lo que es fundamental problematizar y reflexionar respecto a qué noción de ciudadanía sustenta estos lineamientos y qué tipo de ciudadano se configura al momento de pensar en los migrantes regionales.

En primer lugar, al no existir un cuestionamiento a la perspectiva de la gobernabilidad migratoria - sino más bien esta es compartida y compatible con los lineamientos estudiados-, se piensa en un tipo de ciudadano vinculado o que necesariamente se encuentre inserto en los mercados de trabajo. El énfasis en la defensa de los derechos humanos y la construcción de proyectos políticos en clave de ciudadanía convive y no es contradictoria con los acuerdos de residencia sustentados en una lógica economicista e instrumentalista. Los proyectos de construcción de una ciudadanía regional, si bien en diferentes grados y con matices significativos, mantienen una visión que reduce el abordaje del fenómeno migratorio al desplazamiento de fuerza de trabajo debido a que el eje continúa basándose en un instrumentalismo laborista propio de las políticas de gestión de las migraciones, políticas que persiguen canalizar y regular la migración basado en las necesidades de los mercados de trabajo y no las migraciones en sí mismas. Los criterios para solicitar la residencia colocan a los migrantes en la obligatoriedad de justificar su derecho a permanecer a partir de demostrar su utilidad económica en relación con su "adecuada" inserción en dichos mercados. En este último punto, la propuesta de construcción de ciudadanía de Unasur habilita un espacio de debate respecto a las limitaciones que se señalaron para los casos de la cAN y Mercosur ya que, a pesar de que es un planteo incipiente, problematiza el requisito de poseer un certificado de medios de vida lícitos, que vincula de forma directa migración, trabajo y residencia. 
En segundo lugar, los lineamientos se reducen a garantizar ciertos derechos sociales, económicos y laborales pero no necesariamente promueven la membresía política de los migrantes, ni tampoco abordan las dimensiones culturales implicadas en los procesos de integración. De este modo, a pesar de que se comienzan a mencionar y debatir derechos políticos y de participación para los migrantes regionales, se encuentran fuertes limitantes en su tratamiento debido a que no se avanza en mecanismos concretos que permitan la efectiva ampliación de derechos políticos, siendo prácticamente nulos los avances en este sentido. Una situación similar se observa en el tratamiento de la dimensión cultural, la cual, al ser abordada desde las perspectivas afines al pluralismo cultural, se limita a políticas de reconocimiento cultural que niegan el conflicto y los procesos de jerarquización y exclusión social que se producen a partir de la identidad cultural de los migrantes.

Es así que, la categoría de ciudadanía en los lineamientos de la can, Mercosur y Unasur, a pesar de ampliar algunos derechos para los migrantes regionales, se limita a establecer mecanismos de regularización ya que, a fin de cuentas, se considera ciudadanos regionales a aquellos migrantes que logran regularizar su situación migratoria. Pero el problema reside en que englobar los mecanismos de regularización migratoria bajo la categoría de ciudadanía supone establecer una multiplicidad de estatus legales bajo la figura legal de ciudadano, esto debido a que se crean una serie de distinciones administrativas al interior de los espacios regionales y de los mercados laborales nacionales (Mezzadra y Neilson, 2016). Al mismo tiempo que, mediante la categoría de ciudadanía, se instrumentaliza un modo diferencial de integración que jerarquiza y segmenta el acceso a derechos laborales, sociales, culturales, económicos y políticos (Varela, 2015).

En tercer lugar, si bien existen diferencias significativas en relación con los lineamientos de Mercosur y la Comunidad Andina de Naciones y un nulo tratamiento en Unasur, ninguno de los proyectos de construcción de ciudadanía regional plantea eliminar la exigencia de no poseer antecedentes penales, judiciales y policiales, reforzando y sosteniendo las narrativas de securitización que pesan sobre los migrantes regionales.

Por último, se advierte que en los lineamientos estudiados no se trasciende el marco nacional ya que si bien establecen el compromiso de garantizar derechos en un espacio de alcance regional, son los Estados nacionales las únicas estructuras institucionales ante las que se puede apelar, no solo para solicitar y tramitar la residencia, sino también para reclamar y demandar la vulneración o violación de derechos. Ninguno de los organismos de integración regional posee instituciones o instancias de carácter supranacional, por lo que los migrantes regionales se encuentran supeditados a leyes y procedimientos nacionales.

\section{Referencias}

Bulcourf, P. y Cardozo, N. (2008). ¿Por qué comparar políticas públicas? Política Comparada, 1- 49.

Comunidad Andina de Naciones (CAN). (2008). Primer Foro Andino de Migraciones. Quito, Ecuador: Autor. 
Comunidad Andina de Naciones (CAN). (2009). Segundo Foro Andino de Migraciones. Lima, Perú: Autor.

Comunidad Andina de Naciones (CAN). (2012). Tercer Foro Andino de Migraciones. Quito, Ecuador: Autor.

Comunidad Andina de Naciones (CAN). (2013). Cuarto Foro Andino de Migraciones. Bogotá, Colombia: Autor.

Comunidad Andina de Naciones (CAN). (s.f.). Recuperado de www.comunidadandina. org.

Devoto, F. (2004). La historia comparada entre el método y la práctica. Un itinerario historiográfico. Prismas Revista de Historia Intelectual, (8), 229-243.

Domenech, E. (2007). Migraciones contemporáneas y pluralismo cultural en la Argentina: El discurso oficial y las prácticas escolares. En Cartografias da Imigracão: Intercultutalidade e Políticas Públicas (pp. 19-45). Porto Alegre, Brasil: Universidad Federal do Rio Grande do Sul.

Domenech, E. (2008). La ciudadanización de la política migratoria en la región sudamericana: vicisitudes de la agenda global. En S. Novick (Comp.), Las migraciones en América Latina (pp. 53-71). Buenos Aires, Argentina: Catálogos, Clacso.

Domenech, E. (2009). La visión estatal sobre las migraciones en la Argentina reciente. De la retórica de la exclusión a la retórica de la inclusión. En E. Domenech (Coord.), Migración y política: El Estado interrogado. Procesos actuales en Argentina y Sudamérica (pp. 21-69). Córdoba, Argentina: Editorial de la Universidad Nacional de Córdoba.

Domenech, E. (2013). Las migraciones son como el agua: Hacia la instauración de políticas de control con rostro humano. La gobernabilidad migratoria en la Argentina. POLIS Revista Latinoamérica, (35), 2-17.

Domenech, E. y Magliano, M. J. (2008). Migración e inmigrantes en la Argentina reciente: políticas y discursos de exclusión/inclusión. En Pobreza, exclusión social y discriminación étnico-racial en América Latina y el Caribe (pp. 423-448). Bogotá, Colombia: Siglo del Hombre, Clacso.

Estupiñán, M. L. (2013). Acerca de la gobernanza y la gestión migratoria. Crítica del papel de la orm en el gobierno de la migración internacional y regional. Serie Documentos de Trabajo (núm. 33). Buenos Aires, Argentina: Clacso.

Geiger, M. y Pécoud, A. (2012). The New Politics of International Mobility. Migration Management and its Discontents. Basingstoke, Reino Unido: Palgrave Macmillian.

Mármora, L. (2010). Modelos de gobernabilidad migratoria. La perspectiva política en América del Sur. Revista Interdisciplinar da Mobilidade Humana, 18(35), 71-92.

Mercado Común del Sur (Mercosur). (s.f.a). Cartilla de la Ciudadanía del Mercosur. Compilación de Normas relacionadas con el ciudadano y la ciudadana del Mercosur. Recuperado de http://www.cartillaciudadania.mercosur.int

Mercado Común del Sur (Mercosur). (s.f.b). Memoria Institucional del Foro Especializado Migratorio del Mercosur y Estados Asociados, Reunión de Ministros del Interior. Período 1997-2014.

Mercado Común del Sur (Mercosur). (s.f.c). Recuperado de www.mercosur.int.

Mezzadra, S. (2005). Derecho de Fuga. Migraciones, ciudadanía y globalización. España: Traficantes de Sueños. 
Mezzadra, S. y Neilson, B. (2016). La frontera como método, o, la multiplicación del trabajo. Buenos Aires, Argentina: Tinta Limón.

Ramírez, J. (2016). Hacia el Sur. La construcción de la ciudadanía suramericana y la movilidad intrarregional. Ecuador: CELAG.

Romano, S. (2009). Integración económica, desarrollo y migraciones en el Mercosur. Una aproximación crítica. En E. Domenech (Coord.), Migración y política. El Estado interrogado. Procesos actuales en Argentina y Sudamérica (pp. 257-300). Córdoba, Argentina: Editorial de la Universidad Nacional de Córdoba.

Sanahuja, J. A. (2012). Regionalismo post-liberal y multilateralismo en Sudamérica: El caso de unasur. En A. Serbin, L. Martínez y H. Ramanzini (Coords.), El Regionalismo Post-liberal en América Latina y el Caribe: Nuevos actores, nuevos temas y nuevos desafios. Anuario de la Integración Regional de América Latina y el Gran Caribe (pp. 19-72). Buenos Aires, Argentina: Coordinadora Regional de Investigaciones Económicas y Sociales.

Santi, S. (2011). El nuevo orden migratorio global: El papel de la Organización Internacional para las Migraciones (OIM) en la Conferencia Sudamericana sobre Migraciones. Trabajo presentado en el $V$ Encuentro del Centro de Reflexión en Política Internacional (CERPI)/ III Jornadas del Centro de Estudios Sudamericanos (CENSUD), Universidad Nacional de La Plata, Argentina.

Serbin, A. (2010). Regionalismo y soberanía nacional en América Latina: Los nuevos desafios. (Documentos CRIEs 15). Buenos Aires, Argentina: CRIES, Nueva Sociedad.

Unión de Naciones Suramericanas (Unasur). (2006). Declaración de Cochabamba: Colocando la Piedra Fundamental para una Unión Sudamericana. II Cumbre de Jefes de Estado de la Comunidad Sudamericana de Naciones, Cochabamba, Bolivia.

Unión de Naciones Suramericanas (Unasur). (2013). Información sobre las actividades del Grupo de Trabajo sobre Ciudadanía Suramericana (GTCS). Autor.

Unión de Naciones Suramericanas (Unasur). (2016). Informe de la reunión presencial del GTCS. Quito, Ecuador: Autor.

Unión de Naciones Suramericanas (Unasur). (s.f.). Recuperado de www.unasursg.org

Varela, A. (2015). La ciudadanía instituida en la era de la resistencia: El movimiento migrante en Barcelona como agente de nuevos discursos sobre la ciudadanía. En B. Bolaños (Coord.), Biopolítica y Migración. El eslabón perdido de la globalización (pp. 261308). México: Universidad Autónoma Metropolitana, Unidad Cuajimalpa.

Vior, E. (2013). Nacionalidad y supranacionalidad en la construcción de la ciudadanía sudamericana, publicación presentada en el panel "Ciudadanía sudamericana". XI Congreso Argentino de Ciencia Política, Universidad de Entre Ríos, Argentina.

\section{Material legislativo}

Acuerdo $\mathrm{N}^{\circ}$ 14/02 Residencia para nacionales de los Estados partes del Mercosur, Bolivia y Chile. DEc. CMC N 28/02, Mercado Común del Sur (Mercosur) (2002).

Decisión 1343 Estatuto Andino de Movilidad Humana. Parlamento Andino, Comunidad Andina de Naciones (2015).

Decisión 545 Instrumento Andino de Migración Laboral, Comunidad Andina de Naciones (2003). 
Decisión No 8/2012. Aprobada mediante Resolución N²7/2012 por el Consejo de Ministras y Ministros de Relaciones Exteriores de Unasur, Lima, Perú (2012).

Estatuto de la Ciudadanía del Mercosur. Plan de Acción. DEc. CMC N 64/10 (2010).

Informe Conceptual sobre Ciudadanía Sudamericana. Grupo de Trabajo sobre Ciudadanía Sudamericana (GTCS). Aprobado mediante Resolución N ${ }^{\circ}$ 14/2014 por el Consejo de Ministras y Ministros de Relaciones Exteriores de Unasur, Guayaquil, Ecuador (2014).

Tratado Constitutivo de la Unión de Naciones Sudamericanas (2011).

\begin{abstract}
María Gabriela Rho
Argentina. Licenciada en Historia por la Facultad de Filosofía y Humanidades de la Universidad Nacional de Córdoba (FFYH-UNC). Becaria doctoral del Centro de Investigaciones y Estudios sobre Cultura y Sociedad CIECS (Conicet y UNC). Doctorando en Historia en el Doctorado de Historia de la FFyH-UNC. Líneas de investigación: políticas migratorias, estudios críticos de migración y frontera, derechos humanos y ciudadanía. Una publicación reciente es: Rho, M. G. (2016). ¿Ciclo pos-neoliberal? Una aproximación histórico-conceptual a la crisis del neoliberalismo en Sudamérica, Red de Intercátedras de Historia de América Latina Contemporánea (RIHALC), 3(5), 122-132.
\end{abstract}

